\title{
Expression of B-cell-associated genes in peripheral blood mononuclear cells of patients with symptomatic pulmonary embolism
}

\author{
WEI LV ${ }^{1 *}$, QIANGLIN DUAN ${ }^{1 *}$, LEMIN WANG $^{1}$, ZHU GONG $^{1}$, FAN YANG $^{2}$ and YANLI SONG ${ }^{1}$ \\ Departments of ${ }^{1}$ Cardiology and ${ }^{2}$ Laboratory Medicine, Tongji Hospital, Tongji University, Shanghai 200065, P.R. China
}

Received February 11, 2014; Accepted October 24, 2014

DOI: $10.3892 / \mathrm{mmr} .2014 .2978$

\begin{abstract}
The aim of the present study was to identify differentially expressed B-cell-associated genes in peripheral blood mononuclear cells and investigate the gene expression characteristics of the different stages of B-cell activation. A total of 20 patients with pulmonary embolisms (PE) and 20 age- and gender-matched controls were enrolled in the present study. Human complementary DNA microarray analysis was used in order to detect the differential expression of B-cell-associated genes between the PE and control groups. Messenger (m)RNA expression was detected for 82 genes involved in B-cell activation. The results showed that PE patients exhibited significantly increased expression levels of the B-cell receptor genes LYN, CD22, SYK, BTK, PTPRC and NFAM1, whereas expression levels of FYN, FCRL4 and LAX1 were significantly decreased compared to those of the control group. Expression levels of T-cell-dependent B-cell-activation genes, including EMR2, TNFSF9, CD86, ICOSLG, CD37 and CD97, were significantly upregulated in PE patients, whereas SPN mRNA expression was significantly downregulated compared with those of the control group. LILRA1 and TLR9 T cell-independent B-cell activation mRNAs were significantly upregulated in PE patients compared with those of the control group. In addition, the expression levels of B-cell-activation regulator genes, including CR1, LILRB4 and VAV1, were significantly increased, whereas SLAMF7 expression levels were significantly decreased in PE patients compared with those of the control group. Furthermore, the expression levels of B-cell-activation-associated cytokine genes demonstrated a significant upregulation of LTA and IL10 and downregulation of L1A, IFNA5, IFNA6, IFNA8, IFNA14, IL2, IL13 and IFNG in PE patients compared to those of the control group. In conclusion, the differential gene expression at
\end{abstract}

Correspondence to: Mr. Lemin Wang, Department of Cardiology, Tongji Hospital, Tongji University, 389 Xincun Road, Putuo, Shanghai 200065, P.R. China

E-mail:wanglemin@tongji.edu.cn

*Contributed equally

Key words: pulmonary embolism, B cell, messenger RNA different stages of B-cell activation between healthy controls and PE patients indicated that B-cell function was reduced or disorganized in patients with symptomatic PE.

\section{Introduction}

Venous thromboembolism (VTE) is a disease which consists of deep vein thrombosis (DVT) and pulmonary thromboembolism (PTE) (1). Pulmonary embolism (PE), which can be subdivided into acute pulmonary embolisms (APE) and chronic thromboembolic pulmonary hypertension (CTEPH), is a global health problem with high incidence, mortality and misdiagnosis rates (2). The risk factors for VTE include environmental and hereditary influences; however, environmental factors are more commonly linked to the disease (3). Nine editions of guidelines for the prevention, diagnosis and treatment of VTE in surgical patients have been published by The American College of Chest Physicians since 1995 (4,5); however, the incidence rate of symptomatic VTE has not been reduced (6). One possible explanation for this may be that the pathogenesis of VTE has not yet been fully elucidated.

Smeeth et al (7) reported an association between acute infections and the increased risk of VTE. Another study reported that VTEs were identified in multiple organ samples, including the lungs, spleen, pancreas, kidneys and adrenal glands of patients that succumbed to severe acute respiratory syndrome (8). In addition, a previous study demonstrated that messenger (m)RNA expression of genes associated with natural killer (NK) and $\mathrm{T}$ cells were significantly downregulated in patients with symptomatic PE (9), and cellular immune function was decreased in patients with acute PE and CTEPH $(10,11)$. These studies indicated that the occurrence and progress of symptomatic PE were closely associated with the innate and adaptive immune system.

The innate and adaptive immune system influence and interact with each other. T and B cells, as key components of the adaptive immune system, co-operate in order to form the immune response to non-self antigens (12). A previous study reported that mRNA expression of CD19 was downregulated in patients with symptomatic VTE (13). The present study aimed to investigate the expression of a wide range of B-cell-associated genes involved in B-cell activation using human complementary (c)DNA microarray analysis in order to identify differential gene expression at different stages of B-cell activation between PE patients and healthy controls. 


\section{Materials and methods}

Patients. A total of 20 PE patients were enrolled in the present study from Tongji Hospital of Tongji University (Shanghai, China) between 2007-2008. PE patients were required to meet any two of the following three criteria prior to enrollment in the study: i) a selective pulmonary angiography showing pulmonary artery obstruction or filling defects; ii) a lung ventilation/perfusion scan showing single or multiple blood perfusion defects, normal or abnormal ventilation and a ventilation/perfusion mismatch; and iii) a clinical diagnosis indicating that there are risk factors for PE and other cardiovascular diseases, which may have been demonstrated through clinical performance, an electrocardiogram and chest film, arterial blood gas analysis indicating hypoxemia and hypocapnia, D-dimer detection, echocardiography or chest computed tomography. A total of 20 healthy volunteers were enrolled in the present study as controls. The groups were divided as follows: PE group, 20 patients (11 males and nine females; mean age, $70 \pm 14$ and range, 44-89) which included three cases of CTEPH; control group, 20 age- and gender-matched volunteers (11 males and nine females; mean age, $70 \pm 14$ and range, 44-91) who passed health examinations for PE, DVT, arterial thrombosis and congenital coagulation abnormalities. There was no statistically significant difference in age between the two groups $(\mathrm{P}>0.05)$. The present clinical trial was approved by the Ethics Committee of Tongji University and written informed consent was obtained from all PE patients and healthy volunteers.

Gene expression profiling. Agilent G4112A Whole Human Genome Oligo Microarrays were obtained from Agilent Technologies (Santa Clara, CA, USA). Microarrays were composed of 44,290 spots, including 41,675 genes or transcripts, 314 negative control spots, 1,924 positive control spots and 359 blank spots. The functions of more than $70 \%$ of the genes in the microarray had been previously elucidated. Blood samples from all participants were subjected to microarray analysis.

Total RNA isolation. EDTA-anti-coagulated peripheral fasted blood samples $(5 \mathrm{ml})$ were drawn from PE patients and healthy volunteers immediately following admittance to the hospital. Mononuclear cells were obtained from the samples using density gradient centrifugation at 7,000 $\mathrm{x} g$ for $30 \mathrm{~min}$ with Ficoll solution (Agilent Technologies) and the remaining red blood cells were ablated using erythrocyte lysis buffer (Qiagen, Limburg, Netherlands). Total RNA was extracted from mononuclear cells using TRIzol ${ }^{\circledR}$ reagent (Invitrogen Life Technologies, Carlsbad, CA, USA) and then purified using a Qiagen RNeasy column (Qiagen) according to the manufacturer's instructions. Isolated total RNA was evaluated and quantified using a Nanodrop ND-1000 spectrophotometer (Nanodrop Technology, Inc., Wilmington, DE, USA).

Detection of gene expression. Reverse transcription of $\sim 1 \mu \mathrm{g}$ total RNA was performed using an Agilent Low RNA Input Linear Amplification kit (Agilent Technologies) in order to produce double-stranded cDNA. Following purification, an Agilent Low RNA Input Linear Amplification kit (Agilent
Technologies) was used to perform in vitro amplification and modified $\alpha$-D-glucose-1-phosphate uridylyltransferase [UTP; aaUTP, 5-(3-aminoallyl)-UTP; Agilent Technologies] was used to replace UTP. The integrated aaUTP interacted with cyanine-3- $N$-hydroxysuccinimide ester (Agilent Technologies) forming fluorescent products, which were then used for hybridization. The integration rate of fluorescence was determined using a NanodropND-1000 spectrophotometer. The hybridization mixture was then prepared using an Agilent Oligonucleotide Microarray in situ Hybridization Plus kit (Agilent Technologies). Fluorescent products ( $750 \mathrm{ng})$ were fragmented at $60^{\circ} \mathrm{C}$ and hybridization was conducted using Human Whole-Genome 60-mer oligo-chips (G4112F; Agilent Technologies) at $60^{\circ} \mathrm{C}$ for $17 \mathrm{~h}$ at $600 \mathrm{x} \mathrm{g}$. Following hybridization, the chips were washed with Agilent Gene Expression Wash-Buffer (Agilent Technologies) according to manufacturer's instructions. The original signals were then obtained using an Agilent scanner (G2655AA; Agilent Technologies) and Feature Extraction software (GeenSpring GX; Agilent Technologies). The standardization of original signals was performed using the robust multiarray averaging method; standardized signal values were then used for the screening of differentially expressed genes.

Quantitative polymerase chain reaction $(q P C R)$. Three genes were selected at random from the genes with differential expressions between the two groups, and then subjected to qPCR. Relative gene expression levels were normalized to that of GAPDH $\left(2^{-\Delta \Delta C t}\right)$. Differences in gene expression between the two groups were analyzed using a standardized melting curve and the $2^{-\Delta \Delta \mathrm{Ct}}$ method. Results from qPCR were found to be consistent with microarray analysis.

Statistical analysis. Statistical tests were performed using SPSS 17.0 software (International Business Machines, Armonk, NY, USA). Values are presented as the mean \pm standard deviation. Independent-Samples t-tests were used to compare mRNA levels in samples from patients with symptomatic PE and controls. Prior to t-testing, tests for equality of variances were performed in order to test the homogeneity of variance between two independent samples, if variances were not equal, the results of t-tests would be corrected accordingly. $\mathrm{P}<0.05$ was considered to indicate a statistically significant difference between values.

\section{Results}

Expression of B-cell receptor-associated B-cell activation genes. A total of $22 \mathrm{~B}$ cell receptor-associated B-cell activation genes were detected in the peripheral blood mononuclear cells (PBMCs) of patients with PE and the control group (Table I and Fig. 1). The results showed that gene expression levels of LYN, CD22, SYK, BTK, PTPRC and NFAM1 were significantly upregulated in PE patients compared to those of the control group $(\mathrm{P}<0.05)$. However, expression levels of FYN, FCRL4 and LAX1 were significantly lower in PBMCs from $\mathrm{PE}$ patients compared to those in the control group $(\mathrm{P}<0.05)$.

Expression of genes associated with T cell-dependent B-cell activation. A total of 22 genes associated with T cell-dependent 
Table I. Expression of genes associated with B cell receptor-mediated B-cell activation.

\begin{tabular}{lccc}
\hline Symbol & PE & $\begin{array}{c}\text { Control } \\
(\mathrm{X} \pm \mathrm{SD})\end{array}$ & $\mathrm{P}$ \\
\hline BLK & $12.50 \pm 0.80$ & $12.55 \pm 0.69$ & 0.823 \\
BLNK & $10.76 \pm 0.66$ & $10.74 \pm 0.57$ & 0.909 \\
BTK & $13.77 \pm 0.52$ & $13.30 \pm 0.51$ & $0.007^{\mathrm{a}}$ \\
CD5 & $13.68 \pm 0.73$ & $13.58 \pm 0.65$ & 0.653 \\
CD19 & $13.93 \pm 0.95$ & $14.02 \pm 0.69$ & 0.736 \\
CR2 & $10.34 \pm 0.68$ & $10.18 \pm 0.61$ & 0.430 \\
PTPRC & $17.33 \pm 0.37$ & $17.01 \pm 0.25$ & $0.003^{\mathrm{a}}$ \\
CD79A & $14.76 \pm 0.79$ & $14.70 \pm 0.70$ & 0.809 \\
CD79B & $13.20 \pm 0.68$ & $13.19 \pm 0.49$ & 0.967 \\
CD81 & $15.17 \pm 0.53$ & $15.20 \pm 0.44$ & 0.851 \\
FCRL1 & $11.28 \pm 0.80$ & $11.03 \pm 0.85$ & 0.357 \\
FCRL2 & $10.83 \pm 0.79$ & $10.64 \pm 0.62$ & 0.409 \\
FCRL3 & $11.18 \pm 0.74$ & $11.40 \pm 0.69$ & 0.344 \\
FCRL4 & $4.29 \pm 0.38$ & $4.77 \pm 0.61$ & $0.004^{\mathrm{a}}$ \\
FCRL5 & $11.07 \pm 0.93$ & $11.34 \pm 0.87$ & 0.346 \\
FYN & $15.79 \pm 0.32$ & $16.01 \pm 0.31$ & $0.033^{\mathrm{a}}$ \\
LAX1 & $12.14 \pm 0.66$ & $12.68 \pm 0.33$ & $0.002^{\mathrm{a}}$ \\
LYN & $17.47 \pm 0.46$ & $17.06 \pm 0.57$ & $0.016^{\mathrm{a}}$ \\
NFAM1 & $10.63 \pm 0.68$ & $9.69 \pm 0.69$ & $0.000^{\mathrm{a}}$ \\
PIR & $6.32 \pm 0.74$ & $6.07 \pm 0.64$ & 0.257 \\
CD22 & $10.41 \pm 1.17$ & $9.51 \pm 1.23$ & $0.023^{\mathrm{a}}$ \\
SYK & $15.78 \pm 0.46$ & $15.44 \pm 0.34$ & $0.011^{\mathrm{a}}$ \\
\hline & & & \\
\hline
\end{tabular}

${ }^{\mathrm{a}} \mathrm{P}<0.05$ vs. control group. $\mathrm{PE}$, pulmonary embolism; $\mathrm{P}, \mathrm{P}$-value; $\mathrm{X}$, mean; SD, standard deviation.

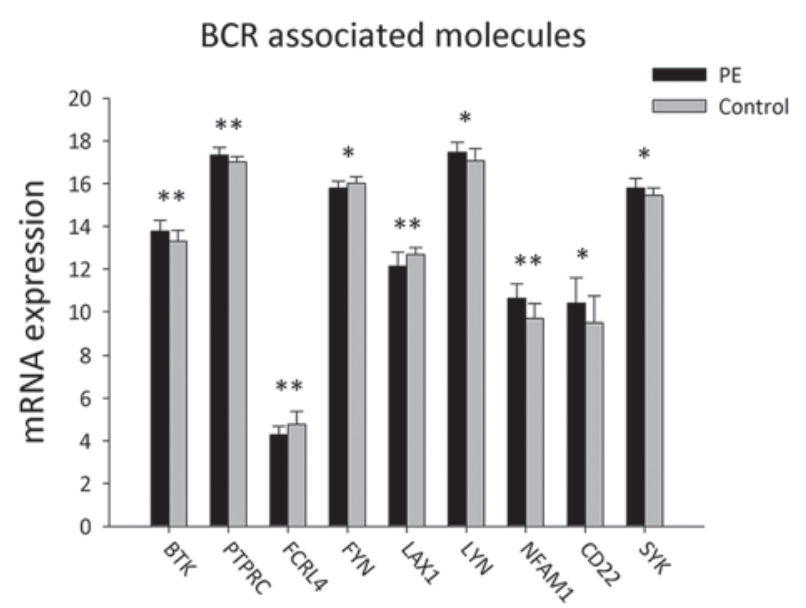

Figure 1. Differential expression of genes associated with BCR-mediated B-cell activation in the peripheral blood mononuclear cells of PE patients and healthy controls. ${ }^{*} \mathrm{P}<0.05$ between $\mathrm{PE}$ and control groups, ${ }^{* *} \mathrm{P}<0.01$ between $\mathrm{PE}$ and control groups. BCR, B cell receptor; PE, pulmonary embolism; mRNA, messenger RNA.

B-cell activation were detected in the PBMCs of PE patients and healthy volunteers (Table II and Fig. 2). In PE patients, the mRNA expression of EMR2, TNFSF9, CD86, ICOSLG, CD37 and CD97 were significantly increased compared to those of
Table II. Expression of genes associated with T cell-dependent B-cell activation.

\begin{tabular}{lrrc}
\hline & \multicolumn{1}{c}{ PE } & $\begin{array}{c}\text { Control } \\
(\mathrm{X} \pm \mathrm{SD})\end{array}$ & $\mathrm{P}$ \\
\hline Symbol & $(\mathrm{X} \pm \mathrm{SD})$ & $8.35 \pm 0.68$ & $0.002^{\mathrm{a}}$ \\
TNFSF9 & $9.04 \pm 0.60$ & $5.68 \pm 0.78$ & 0.616 \\
CD80 & $5.56 \pm 0.76$ & $10.43 \pm 0.64$ & $0.001^{\mathrm{a}}$ \\
ICD86 & $11.14 \pm 0.67$ & $9.45 \pm 0.47$ & $0.008^{\mathrm{a}}$ \\
CD276 & $9.81 \pm 0.33$ & $5.11 \pm 1.15$ & 0.562 \\
BTLA & $4.92 \pm 0.94$ & $10.07 \pm 0.81$ & 0.865 \\
CD28 & $10.03 \pm 0.69$ & $11.70 \pm 0.57$ & 0.301 \\
CD37 & $11.46 \pm 0.85$ & $15.49 \pm 0.69$ & $0.001^{\mathrm{a}}$ \\
CD40 & $16.28 \pm 0.73$ & $9.73 \pm 0.58$ & 0.053 \\
SPN $(\mathrm{CD} 43)$ & $14.30 \pm 0.75$ & $14.79 \pm 0.49$ & $0.020^{\mathrm{a}}$ \\
CD72 & $11.14 \pm 0.79$ & $11.35 \pm 0.59$ & 0.349 \\
CD84 & $12.27 \pm 0.57$ & $11.91 \pm 0.67$ & 0.069 \\
CD97 & $16.83 \pm 0.85$ & $16.14 \pm 0.54$ & $0.004^{\mathrm{a}}$ \\
LY9 & $12.52 \pm 0.61$ & $12.65 \pm 0.50$ & 0.451 \\
CTLA4 & $7.51 \pm 1.00$ & $7.59 \pm 0.62$ & 0.772 \\
CD226 & $12.14 \pm 0.71$ & $12.04 \pm 0.39$ & 0.598 \\
EMR2 & $14.73 \pm 0.77$ & $14.18 \pm 0.53$ & $0.011^{\mathrm{a}}$ \\
MS4A1 & $13.01 \pm 0.97$ & $13.11 \pm 0.73$ & 0.708 \\
TNFSF4 & $11.82 \pm 0.67$ & $11.56 \pm 0.51$ & 0.171 \\
PDCD1 & $11.50 \pm 0.56$ & $11.86 \pm 0.58$ & 0.052 \\
SLAMF1 & $11.29 \pm 0.53$ & $11.53 \pm 0.55$ & 0.158 \\
\hline
\end{tabular}

${ }^{\mathrm{a}} \mathrm{P}<0.05$ vs. control group. $\mathrm{PE}$, pulmonary embolism; $\mathrm{P}, \mathrm{P}$-value; $\mathrm{X}$, mean; SD, standard deviation.

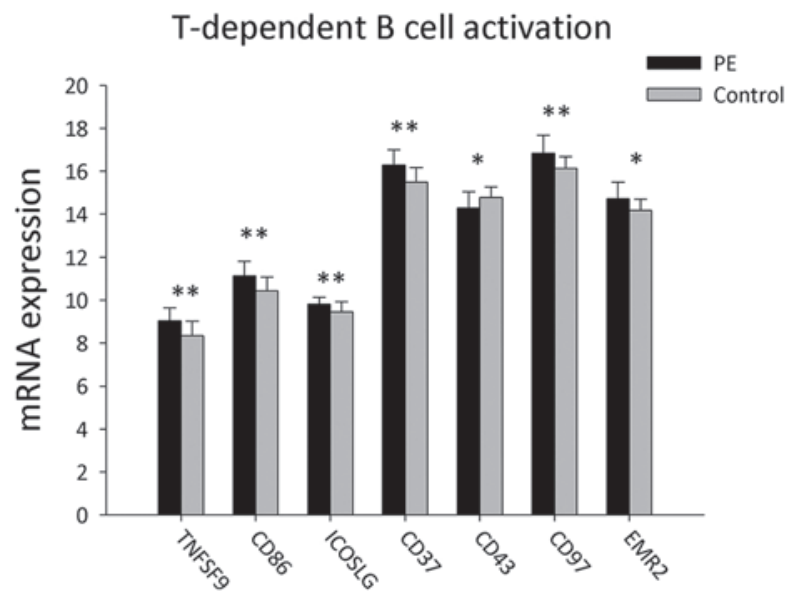

Figure 2. Differential expression of genes associated with T cell-dependent B-cell activation in the peripheral blood mononuclear cells of PE patients and healthy controls. ${ }^{*} \mathrm{P}<0.05$ between $\mathrm{PE}$ and control groups, ${ }^{* *} \mathrm{P}<0.01$ between $\mathrm{PE}$ and control groups. PE, pulmonary embolism; mRNA, messenger RNA.

the healthy volunteers $(\mathrm{P}<0.05)$, whereas SPN $(\mathrm{CD} 43)$ mRNA was significantly decreased $(\mathrm{P}<0.05)$.

Expression of genes associated with T cell-independent B-cell activation. The results showed that mRNA expressions of seven 
Table III. Expression of genes associated with T cell-independent B-cell activation.

\begin{tabular}{lrrr}
\hline Symbol & \multicolumn{1}{c}{ PE } & \multicolumn{1}{c}{$\begin{array}{c}\text { Control } \\
(\mathrm{X} \pm \mathrm{SD})\end{array}$} & \multicolumn{1}{c}{$\mathrm{P} \pm \mathrm{SD})$} \\
\hline SLAMF8 & $10.83 \pm 0.91$ & $11.20 \pm 0.58$ & 0.131 \\
UBD & $6.23 \pm 0.82$ & $6.51 \pm 0.50$ & 0.196 \\
FCGR2B & $13.18 \pm 0.54$ & $12.92 \pm 0.62$ & 0.161 \\
FCGR3A & $18.76 \pm 0.38$ & $18.72 \pm 0.39$ & 0.702 \\
LILRA1 & $13.67 \pm 0.81$ & $13.03 \pm 0.52$ & $0.005^{\text {a }}$ \\
CD180 & $11.70 \pm 0.57$ & $11.42 \pm 0.57$ & 0.128 \\
TLR9 & $10.64 \pm 0.66$ & $9.97 \pm 0.46$ & $0.001^{\text {a }}$ \\
\hline
\end{tabular}

${ }^{\mathrm{a}} \mathrm{P}<0.05$ vs. control group. $\mathrm{PE}$, pulmonary embolism; $\mathrm{X}$, mean; $\mathrm{SD}$, standard deviation; P, P-value.

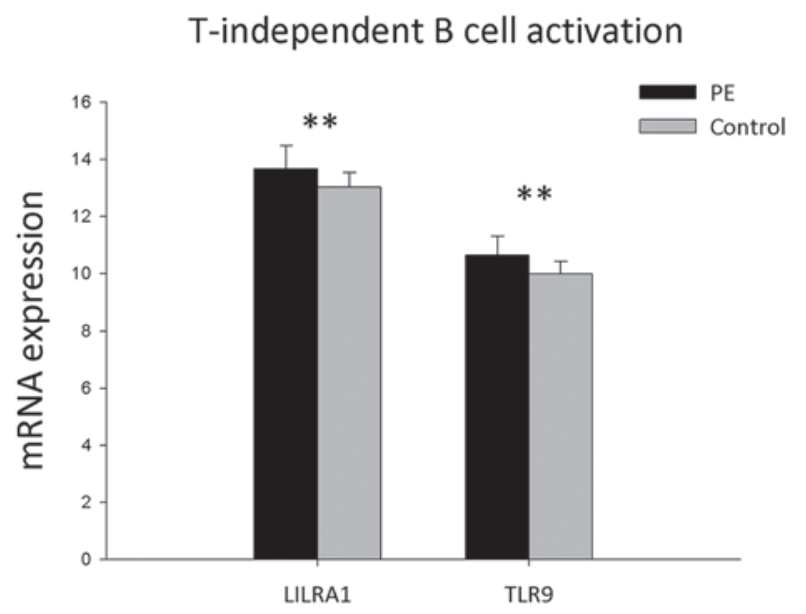

Figure 3. Differential expression of genes associated with T cell-independent B-cell activation in the peripheral blood mononuclear cells of PE patients and healthy controls. " $\mathrm{P}<0.05$ between $\mathrm{PE}$ and control groups, ${ }^{* *} \mathrm{P}<0.01$ between $\mathrm{PE}$ and control groups. PE, pulmonary embolism; mRNA, messenger RNA.

genes associated with $\mathrm{T}$ cell-independent B-cell activation in PBMCs were detected in the PBMCs of the PE and control groups (Table III and Fig. 3). mRNA expression levels of LILRA1 and TLR9 were found to be significantly upregulated in $\mathrm{PE}$ patients compared with those of the control group $(\mathrm{P}<0.05)$.

Expression of B-cell activation regulatory genes. Ten genes involved in the regulation of B-cell activation were identified in the PBMCs of the PE and control groups (Table IV and Fig. 4). The expression levels of genes, including CR1, LILRB4 and VAV1, were significantly increased in PE patients compared to those of the heathy controls $(\mathrm{P}<0.05)$, whereas expression levels of SLAMF7 were significantly decreased in $\mathrm{PE}$ patients $(\mathrm{P}<0.05)$.

Expression levels of B-cell activation-associated cytokine mRNAs. A total of 22 genes of cytokines involved in B-cell activation were detected in the PBMCs of PE patients and healthy controls (Table V and Fig. 5). mRNA expression levels of cytokines, including LTA and IL10, were significantly
Table IV. Gene expression of B-cell activation-associated regulators.

\begin{tabular}{lcrc}
\hline Symbol & $\begin{array}{c}\mathrm{PE} \\
(\mathrm{X} \pm \mathrm{SD})\end{array}$ & $\begin{array}{c}\text { Control } \\
(\mathrm{X} \pm \mathrm{SD})\end{array}$ & $\mathrm{P}$ \\
\hline CR1 (CD35) & $11.98 \pm 1.11$ & $10.63 \pm 0.74$ & $0.000^{\mathrm{a}}$ \\
SLAMF7 & $13.71 \pm 0.83$ & $14.34 \pm 0.44$ & $0.005^{\mathrm{a}}$ \\
IL4I1 & $10.97 \pm 0.80$ & $11.01 \pm 0.65$ & 0.867 \\
LILRB1 & $12.94 \pm 0.70$ & $12.61 \pm 0.94$ & 0.207 \\
LILRB4 & $10.19 \pm 0.66$ & $9.62 \pm 0.52$ & $0.004^{\mathrm{a}}$ \\
LILRB2 & $17.42 \pm 0.50$ & $17.21 \pm 0.38$ & 0.135 \\
LILRB3 & $18.05 \pm 0.47$ & $17.82 \pm 0.42$ & 0.112 \\
LILRA3 & $12.88 \pm 1.60$ & $12.22 \pm 1.30$ & 0.165 \\
LAIR1 & $13.53 \pm 0.52$ & $13.22 \pm 0.50$ & 0.061 \\
VAV1 & $15.93 \pm 0.45$ & $15.49 \pm 0.33$ & $0.001^{\mathrm{a}}$ \\
TLR9 & $10.64 \pm 0.66$ & $9.97 \pm 0.46$ & $0.001^{\mathrm{a}}$ \\
\end{tabular}

${ }^{\mathrm{a}} \mathrm{P}<0.05$ vs. control group. $\mathrm{PE}$, pulmonary embolism; $\mathrm{X}$, mean; SD, standard deviation; P, P-value.

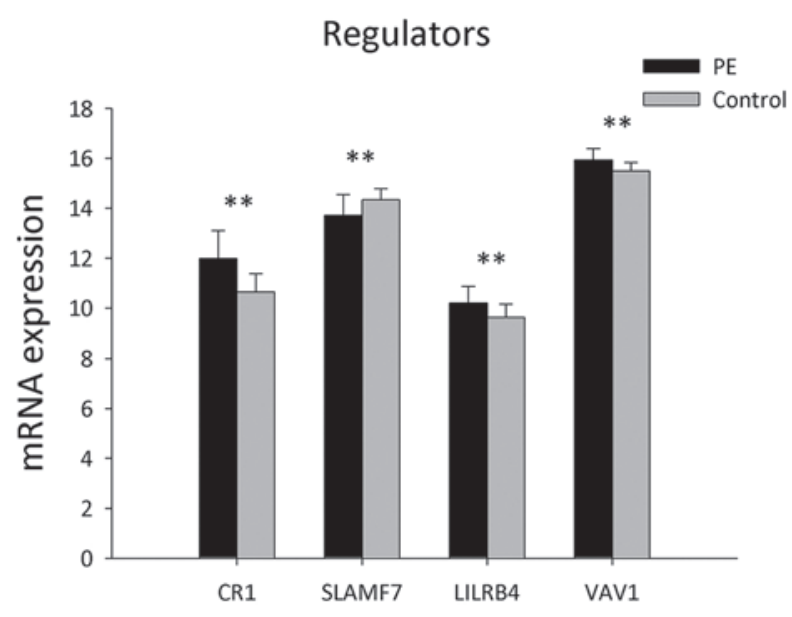

Figure 4. Differential gene expression of B-cell activation-associated regulators in the peripheral blood mononuclear cells of PE patients and healthy controls. "P $<0.05$ between $\mathrm{PE}$ and control groups, ${ }^{* *} \mathrm{P}<0.01$ between $\mathrm{PE}$ and control groups. PE, pulmonary embolism; mRNA, messenger RNA.

upregulated in PE patients compared to those in the control group $(\mathrm{P}<0.05)$, whereas the expressions levels of IL1A, IFNA5, IFNA6, IFNA8, IFNA14, IL2, IL13 and IFNG were significantly decreased in the PBMCs of PE patients $(\mathrm{P}<0.05)$.

\section{Discussion}

A previous study demonstrated that mRNA expression levels of genes associated with $\mathrm{NK}$ and $\mathrm{T}$ cells were downregulated in PE patients (9); in addition, NK-cell and T-cell function as well as mRNA expression of CD19 were reported to be markedly decreased in VTE patients $(10,11,13)$. The present study identified the differential gene expression at different stages of B-cell activation in patients with symptomatic PE. B-cell activation requires two signals, of which the first is dependent on antigen binding to the $\mathrm{B}$-cell antigen receptor. If the antigen 
Table V. Gene expression of B-cell activation-associated cytokines.

\begin{tabular}{lccc}
\hline & \multicolumn{1}{c}{$\mathrm{PE}$} & $\begin{array}{c}\text { Control } \\
(\mathrm{X} \pm \mathrm{SD})\end{array}$ & $\mathrm{P}$ \\
\hline Lymbol & $(\mathrm{X} \pm \mathrm{SD})$ & $4.98 \pm 0.75$ & $0.017^{\mathrm{a}}$ \\
L1B & $4.47 \pm 0.52$ & $14.56 \pm 0.69$ & 0.366 \\
IL2 & $14.79 \pm 0.86$ & $4.99 \pm 0.71$ & $0.006^{\mathrm{a}}$ \\
IL4 & $4.47 \pm 0.38$ & $6.92 \pm 1.51$ & 0.247 \\
IL5 & $6.39 \pm 1.32$ & $4.86 \pm 0.71$ & 0.372 \\
IL6 & $5.10 \pm 0.96$ & $6.55 \pm 0.47$ & 0.575 \\
IL10 & $6.66 \pm 0.71$ & $6.69 \pm 0.48$ & $0.000^{\mathrm{a}}$ \\
IL12A & $7.68 \pm 0.72$ & $7.36 \pm 0.53$ & 0.109 \\
IL12B & $7.03 \pm 0.74$ & $5.39 \pm 0.94$ & 0.847 \\
IL13 & $5.46 \pm 1.48$ & $5.44 \pm 1.09$ & $0.003^{\mathrm{a}}$ \\
TNF & $4.58 \pm 0.55$ & $9.59 \pm 0.95$ & 0.056 \\
LTA & $10.10 \pm 0.66$ & $7.60 \pm 0.47$ & $0.013^{\mathrm{a}}$ \\
IFNA2 & $7.96 \pm 0.39$ & $4.87 \pm 0.71$ & 0.081 \\
IFNA4 & $4.50 \pm 0.60$ & $7.80 \pm 0.47$ & 0.350 \\
IFNA5 & $7.96 \pm 0.54$ & $5.31 \pm 0.70$ & $0.014^{\mathrm{a}}$ \\
IFNA6 & $4.85 \pm 0.40$ & $5.07 \pm 1.41$ & $0.036^{\mathrm{a}}$ \\
IFNA8 & $4.46 \pm 0.54$ & $5.04 \pm 0.86$ & $0.014^{\mathrm{a}}$ \\
IFNA10 & $4.84 \pm 0.89$ & $4.98 \pm 0.73$ & 0.585 \\
IFNA14 & $4.55 \pm 0.53$ & $5.27 \pm 1.07$ & $0.011^{\mathrm{a}}$ \\
IFNA21 & $4.89 \pm 1.60$ & $5.25 \pm 0.90$ & 0.388 \\
IFNB1 & $5.05 \pm 0.94$ & $5.31 \pm 1.17$ & 0.443 \\
IFNG & $9.45 \pm 0.94$ & $10.21 \pm 0.76$ & $0.009^{\mathrm{a}}$ \\
\hline & & & \\
\hline
\end{tabular}

${ }^{\mathrm{a}} \mathrm{P}<0.05$ vs. control group. $\mathrm{PE}$, pulmonary embolism; $\mathrm{X}$, mean; $\mathrm{SD}$, standard deviation; $\mathrm{P}, \mathrm{P}$-value.

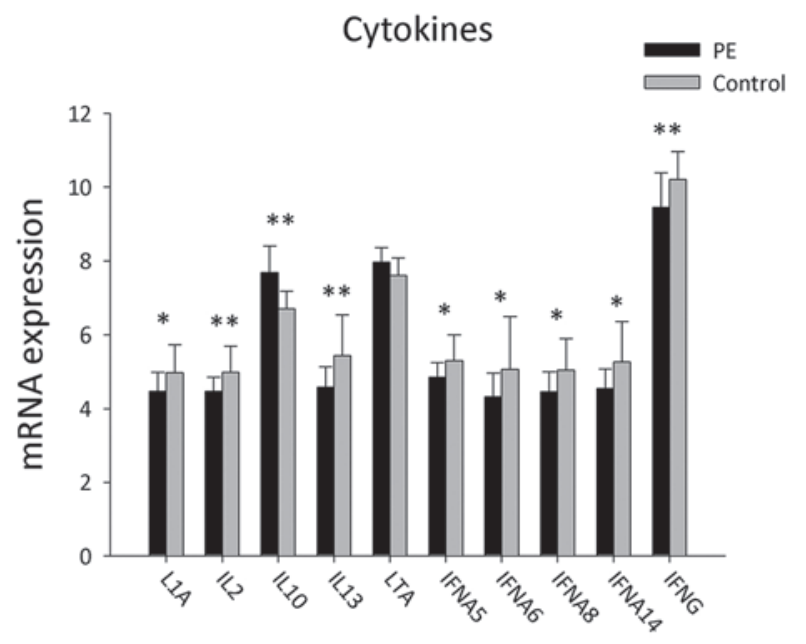

Figure 5. Differential gene expression of B-cell activation-associated cytokines in the peripheral blood mononuclear cells of PE patients and healthy controls. ${ }^{*} \mathrm{P}<0.05$ between $\mathrm{PE}$ and control groups, ${ }^{* *} \mathrm{P}<0.01$ between $\mathrm{PE}$ and control groups. PE, pulmonary embolism; mRNA, messenger RNA.

is thymus-dependent, the second signal is initiated following interactions between T cell and B cell; however, thymus-independent antigens activate the second signal autonomously (14).
The results of the present study showed that the mRNA expression of genes associated with B-cell receptor-mediated B-cell activation, including LYN, CD22, SYK, BTK, PTPRC and NFAM1, were significantly upregulated in PE patients compared with the control group. LYN mediates positive as well as negative roles in B-cell activation (15) and CD22 is an inhibitory co-receptor, which negatively regulates $\mathrm{B}$-cell receptor signaling (16). B-cell activation is initiated by SYK-dependent and -independent signaling cascades (17). In addition, BTK is involved in the regulation of actin dynamics required for antigen processing and presentation in B cells (18). Furthermore, PTPRC-deficient B cells were reported to exhibit impaired proliferation in vitro (19) and the overexpression of NFAM1 resulted in severe impairment of early B-cell development (20).

However, in the present study, the mRNA expression levels of FYN, FCRL4 and LAX1 genes associated with $\mathrm{B}$-cell receptor-mediated B-cell activation were significantly downregulated in PE patients compared with those of the control group. A previous study using FYN-deficient mice demonstrated that FYN had a key role in B-cell activation (21). FCRL4 was found to inhibit B cell receptor signaling in response to chronic antigenic stimulation (22) and LAX was reported to be a negative regulator of B-cell activation, as LAX-deficient B cells were found to be hyper-responsive to B-cell receptor stimulation (23).

These studies indicated that the differential expression of genes associated with B-cell receptor-mediated B-cell activation in PE patients and the control group in the present study may have positively or negatively regulated B-cell activation.

In the present study, the mRNA expression levels of EMR2, TNFSF9, CD86, ICOSLG, CD37 and CD97 genes associated with $\mathrm{T}$ cell-dependent $\mathrm{B}$-cell activation were significantly upregulated in PE patients compared with those of the control group. CD97, EMR2 and CD86 were previously reported to have important roles in the interaction of T cells with B cells $(24,25)$ and the interaction of TNFSF9 and its receptor were shown to positively regulate the humoral immune response (26). The ICOSLG gene encodes the ligand for the inducible co-stimulator receptor, which was reported to have an essential role in T cell-dependent B-cell responses (27). In addition, one study demonstrated that interactions between $\mathrm{T}$ cells and $\mathrm{B}$ cells were significantly impaired in CD37-deficient mice (28).

The present study also demonstrated that expression of the T cell-dependent B-cell activation gene SPN was significantly downregulated in PE patients compared with that of the control group; SPN downregulation has previously been reported to enhance interactions between T cells and B cells (29). These studies therefore indicated that in the present study, the differential gene expression of genes involved in T cell-dependent B-cell activation may enhance interactions between $\mathrm{T}$ cells and $\mathrm{B}$ cells in patients with symptomatic PE compared to those in the healthy controls.

In the present study, seven genes associated with T cell-independent B-cell activation were detected, of which LILRA1 and TLR9 were found to be significantly upregulated in the PBMCs of PE patients compared with those of the control group. A previous study detected LILRA1 mRNA in B cells (30), which was reported to activate cells through associating with the $\mathrm{Fc}$ receptor gamma chain (31); in addition, TLR9 agonists were reported to be involved in the activation of human B cells (32). 
This therefore indicated that $\mathrm{T}$ cell-independent B-cell activation was enhanced in PE patients in the present study.

Increased gene expression of $\mathrm{B}$-cell activation regulators, including CR1, LILRB4 and VAV1, was observed in PE patients compared to that of the control group in the present study. CR1 inhibits B-cell receptor-mediated B-cell activation (33) and LILRB4 negatively regulates the activation of antigen-presenting cells (34); in addition, VAV1-deficient mice were reported to have activation defects in B cells (35). In the present study, mRNA expression of the B-cell activation regulator SLAMF7 was found to be significantly downregulated in PE patients compared to that of the control group. A previous study showed that SLAMF7 increased the proliferation of $B$ cells and autocrine cytokine expression on B cells (36). These previous studies therefore indicated that in the present study, B-cell activation was inhibited in patients with symptomatic PE.

The results of the present study also demonstrated differential gene expression of B-cell activation-associated cytokines. mRNA expression levels of LTA and IL10 were significantly increased in the PBMCs of PE patients compared to those of the healthy controls, whereas expression levels of L1A, IFNA5, IFNA6, IFNA8, IFNA14, IL2, IL13 and IFNG were found to be significantly decreased in PE patients.

In addition to producing antibodies against antigens, $\mathrm{B}$ cells also produce cytokines. IL13 is expressed in activated T cells and has been shown to promote B-cell proliferation as well as the synthesis of Immunoglobulin E (37); furthermore, IFNA/B was reported to induce partial B-cell activation (38). However, IFNG inhibited B-cell differentiation (39). This therefore indicated that the downregulation of cytokine genes impaired B-cell function in PE patients enrolled in the present study.

In conclusion, the present study demonstrated that patients with symptomatic PE exhibited downregulation of genes associated with the B-cell receptor and differential expression of B-cell activation-regulator genes, indicating that B-cell function was inhibited. In addition, the differential expression of genes associated to $\mathrm{T}$ cell-dependent B-cell activation suggested that interactions between $\mathrm{T}$ cells and $\mathrm{B}$ cells were enhanced in PE patients, whereas the upregulation of genes associated with $\mathrm{T}$ cell-independent $\mathrm{B}$-cell activation indicated an increase in T cell-indepenent B-cell activation. Furthermore, the downregulation of B-cell activation-associated cytokines in PE patients indicated that cytokine production by B cells was significantly reduced. These results therefore demonstrated reduced or disorganized B-cell function in patients with symptomatic PE, which is consistent with the cytological results of a previous study (13).

\section{Acknowledgements}

The present study was supported by a grant from the '12th five year' National Science and Technology Supporting Program (no. 2011BAI11B16).

\section{References}

1. Geerts WH, Heit JA, Claggett GP, et al: Prevention of venous thromboembolism. Chest 119 (1 Suppl): 132S-175S, 2001.

2. Heit JA: The epidemiology of venous thromboembolism in the community. Arterioscler Thromb Vasc Biol 28: 370-372, 2008.

3. Martinelli I. Risk factors in venous thromboembolism. Thromb Haemost 86: 395-403, 2001.
4. Clagett GP, Anderson FA Jr, Heit J, Levine MN and Wheeler HB: Prevention of venous thromboembolism. Chest 108 (4 Suppl): 312S-334S, 1995.

5. Kahn SR, Lim W, Dunn AS, et al; American College of Chest Physicians: Prevention of VTE in nonsurgical patients: Antithrombotic therapy and prevention of thrombosis, 9th ed: American College of Chest Physicians evidence-based clinical practice guidelines. Chest 141 (2 Suppl): e195S-e226S, 2012.

6. Shackford SR, Rogers FB, Terrien CM, Bouchard P, Ratliff J and Zubis R: A 10-year analysis of venous thromboembolism on the surgical service: the effect of practice guidelines for prophylaxis. Surgery 144: 3-11, 2008.

7. Smeeth L, Cook C, Thomas S, Hall AJ, Hubbard R and Vallance P: Risk of deep vein thrombosis and pulmonary embolism after acute infection in a community setting. Lancet 367: 1075-1079, 2006.

8. Xiang-Hua Y, Le-Min W, Ai-Bin L, et al: Severe acute respiratory syndrome and venous thromboembolism in multiple organs. Am J Respir Crit Care Med 182: 436-437, 2010.

9. Gong Z, Liang AB, Wang LM, et al: The expression and significance of immunity associated genes mRNA in patients with pulmonary embolism. Chin J Intern Med 48: 666-669, 2009 (In Chinese).

10. Wang L, Song H, Gong Z, Duan Q and Liang A: Acute pulmonary embolism and dysfunction of CD3+ CD8+ T cell immunity. Am J Respir Crit Care Med 184: 1315, 2011.

11. Haoming S, Lemin $\mathrm{W}$, Zhu G, et al: $\mathrm{T}$ cell-mediated immune deficiency or compromise in patients with CTEPH. Am J Respir Crit Care Med 183: 417-418, 2011.

12. O'Leary JG, Goodarzi M, Drayton DL and von Andrian UH: $\mathrm{T}$ cell- and $\mathrm{B}$ cell-independent adaptive immunity mediated by natural killer cells. Nat Immunol 7: 507-516, 2006.

13. Duan Q, Gong Z, Song H, et al: Symptomatic venous thromboembolism is a disease related to infection and immune dysfunction. Int J Med Sci 9: 453-461, 2012.

14. Janeway CA, Jr, Travers P, Walport M and Shlomchik MJ: Immunobiology: The Immune System in Health and Disease. 5th edition. Garland Science, New York, 2001.

15. Gauld SB and Cambier JC: Src-family kinases in B-cell development and signaling. Oncogene 23: 8001-8006, 2004.

16. Nitschke L and Tsubata T: Molecular interactions regulate BCR signal inhibition by CD22 and CD72. Trends Immunol 25: 543-550, 2004.

17. Yokozeki T, Adler K, Lankar D and Bonnerot C: B cell receptor-mediated Syk-independent activation of phosphatidylinositol 3-kinase, Ras, and mitogen-activated protein kinase pathways. J Immunol 171: 1328-1335, 2003.

18. Sharma S, Orlowski G and Song W: Btk regulates B cell receptor-mediated antigen processing and presentation by controlling actin cytoskeleton dynamics in B cells. J Immunol 182: 329-339, 2009.

19. Huntington ND, Xu Y, Puthalakath $\mathrm{H}$, et al: CD45 links the B cell receptor with cell survival and is required for the persistence of germinal centers. Nat Immunol 7: 190-198, 2006.

20. Ohtsuka M, Arase H, Takeuchi A, et al: NFAM1, an immunoreceptor tyrosine-based activation motif-bearing molecule that regulates B cell development and signaling. Proc Natl Acad Sci USA 101: 8126-8131, 2004.

21. Horikawa K, Nishizumi H, Umemori H, Aizawa S, Takatsu K and Yamamoto T: Distinctive roles of Fyn and Lyn in IgD- and IgM-mediated signaling. Int Immunol 11: 1441-1449, 1999.

22. Sohn HW, Krueger PD, Davis RS and Pierce SK: FcRL4 acts as an adaptive to innate molecular switch dampening BCR signaling and enhancing TLR signaling. Blood 118: 6332-6341, 2011.

23. Zhu M, Granillo O, Wen R, et al: Negative regulation of lymphocyte activation by the adaptor protein LAX. J Immunol 174: 5612-5619, 2005.

24. Kwakkenbos MJ, Pouwels W, Matmati M, et al: Expression of the largest CD97 and EMR2 isoforms on leukocytes facilitates a specific interaction with chondroitin sulfate on B cells. J Leukoc Biol 77: 112-119, 2005.

25. McLellan AD, Starling GC, Williams LA, Hock BD and Hart DN: Activation of human peripheral blood dendritic cells induces the CD86 co-stimulatory molecule. Eur J Immunol 25: 2064-2068, 1995.

26. Zhu G, Flies DB, Tamada K, Sun Y, Rodriguez M, Fu YX and Chen L: Progressive depletion of peripheral B lymphocytes in 4-1BB (CD137) ligand/I-Ealpha-transgenic mice. J Immunol 167: 2671-2676, 2001.

27. Mak TW, Shahinian A, Yoshinaga SK, et al: Costimulation through the inducible costimulator ligand is essential for both Thelper and $\mathrm{B}$ cell functions in T cell-dependent B cell responses. Nat Immunol 4: 765-772, 2003. 
28. Knobeloch KP, Wright MD, Ochsenbein AF, et al: Targeted inactivation of the tetraspanin CD37 impairs T-cell-dependent B-cell response under suboptimal costimulatory conditions. Mol Cell Biol 20: 5363-5369, 2000 .

29. Ostberg JR, Dragone LL, Driskell T, et al: Disregulated expression of CD43 (leukosialin, sialophorin) in the B cell lineage leads to immunodeficiency. J Immunol 157: 4876-4884, 1996.

30. Tedla N, Gibson K, McNeil HP, Cosman D, Borges L and Arm JP: The co-expression of activating and inhibitory leukocyte immunoglobulin-like receptors in rheumatoid synovium. Am J Pathol 160: 425-431, 2002.

31. Colonna M, Nakajima H, Navarro F and López-Botet M: A novel family of Ig-like receptors for HLA class I molecules that modulate function of lymphoid and myeloid cells. J Leukoc Biol 66: 375-381, 1999.

32. Hanten JA, Vasilakos JP, Riter CL, et al: Comparison of human B-cell activation by TLR7 and TLR9 agonists. BMC Immunol 9: 39, 2008.

33. Józsi M, Prechl J, Bajtay Z and Erdei A: Complement receptor type $1(\mathrm{CD} 35)$ mediates inhibitory signals in human B lymphocytes. J Immunol 168: 2782-2788, 2002.
34. Cella M, Döhring C, Samaridis J, et al: A novel inhibitory receptor (ILT3) expressed on monocytes, macrophages, and dendritic cells involved in antigen processing. J Exp Med 185: 1743-1751, 1997

35. Fujikawa K, Miletic AV, Alt FW, et al: Vav1/2/3-null mice define an essential role for Vav family proteins in lymphocyte development and activation but a differential requirement in MAPK signaling in T and B cells. J Exp Med 198: 1595-1608, 2003.

36. Lee JK, Mathew SO, Vaidya SV, Kumaresan PR and Mathew PA: CS1 (CR ACC, CD319) induces proliferation and autocrine cytokine expression on human B lymphocytes. J Immunol 179: 4672-4678, 2007.

37. Defrance T, Carayon P, Billian G, et al: Interleukin 13 is a B cell stimulating factor. J Exp Med 179: 135-143, 1994.

38. Braun D, Caramalho I and Demengeot J: IFN-alpha/beta enhances BCR-dependent B cell responses. Int Immunol 14: 411-419, 2002.

39. Abed NS,Chace JH,Fleming AL and Cowdery JS: Interferon-gamma regulation of $\mathrm{B}$ lymphocyte differentiation: activation of $\mathrm{B}$ cells is a prerequisite for IFN-gamma-mediated inhibition of B cell differentiation. Cell Immunol 153: 356-366, 1994. 